\title{
Craniectomia descompressiva para tratamento de hipertensão intracraniana secundária a trauma com posterior cranioplastia: relato de caso
}

\section{Decompressive craniectomy for the treatment of intracranial hypertension secondary to trauma with subsequent cranioplasty: a case report}

\author{
Marcella Markman de Almeida ${ }^{*}$, Caio Atanasio de Morais Ramos ${ }^{1}$, Camila Catharine Pontes Sanches ${ }^{1}$, \\ Karinne Mendes Santos ${ }^{1}$, Maria Áurea de Andrade Borba ${ }^{1}$, Rayana de Albuquerque Guimarães Pimentel' ${ }^{\prime}$, \\ Cícero Pacheco ${ }^{2}$ \\ ${ }^{1}$ Hospital Universitário Oswaldo Cruz, Universidade de Pernambuco, Recife, Brasil \\ ${ }^{2}$ Hospital da Restauração, Recife, Brasil \\ *correspondência: marcella.markman13@gmail.com
}

Recebido: 30/10/2019. Aceito: 12/11/2019.

\begin{abstract}
Resumo
A caixa craniana possui volume constante, estando sujeita a pequenas flutuações de pressão, fisiologicamente situadas entre 5-15 $\mathrm{mmHg}$. Lesões secundárias ao trauma-crânio-encefálico, podem acarretar hipertensão intracraniana. O presente relato descreve o caso de uma mulher com 59 anos, atendida na unidade de trauma do Hospital da Restauração, vítima de queda de própria altura com história de rebaixamento súbito do nível de consciência, seguido de agitação e desorientação. A tomografia computadorizada mostrou hematomas subdural agudo e intraparenquimatoso, com desvio das estruturas da linha média maior que $5 \mathrm{~mm}$, justificando realização de uma craniectomia descompressiva de emergência. A paciente evoluiu estável hemodinamicamente, com déficit hemiparético direito de grau II. Foi então realizada cranioplastia 20 dias após primeira intervenção, de modo a evitar Síndrome do Trefinado e suas complicações.
\end{abstract}

Palavras-chave: craniectomia descompressiva, trauma crânioencefálico, hipertensão intracraniana (HIC).

\begin{abstract}
The skull has constant volume, being sujected to small pressure flotations, fisiologically situated between 5$15 \mathrm{mmHg}$. Traumatic pathologies, such as cranioencephalic trauma (CET) may change those values resulting in diverse haematomas and causing intracranial hypertension (ICH). The present case report describes a case of a 59 year-old-woman with history of fall from her own height and abrupt decrease of conscious followed by agitation and desorientation. The computadorized tomography (CT) showed accute subdural and intracerebral haematoma with midline shift bigger than $5 \mathrm{~mm}$, justifying the emergencial decompressive craniectomy. Cranioplasty was then performed 20 days after the first intervention, avoiding thus Syndrome of the Trephined and its complications.
\end{abstract}

Keywords: decompressive craniectomy, cranioencephalic trauma (CET), intracranial hypertension (ICH)

\section{Introdução}

A caixa craniana de um humano adulto possui volume constante, estando sujeita a pequenas flutuações de pressão, fisiologicamente situadas entre 5-15 mmHg. ${ }^{1}$ No trauma crânio-encefálico (TCE), o paciente pode evoluir com hipertensão intracraniana (HIC). ${ }^{2}$ Em adultos, valores acima de $20 \mathrm{mmHg}$, que duram mais de 10 minutos, devem ser tratados como $\mathrm{HIC}^{1}$, por ser uma emergência médica com risco iminente de vida. Um procedimento cirúrgico que vem sendo cada vez mais empregado nas últimas décadas para reduzir a HIC é a craniectomia descompressiva. ${ }^{3}$ 


\section{Relato de Caso}

Mulher com 59 anos de idade foi atendida na unidade de trauma do Hospital da Restauração, Recife, Brasil. Essa paciente foi vítima de queda da própria altura, com trauma craniano e perda transitória da consciência que foi seguida de agitação e desorientação. No momento do exame neurológico a paciente encontrava-se inconsciente, com escore 6 na escala de coma de Glasgow (ECG) e anisocoria (pupila esquerda maior que a direita). Solicitou-se uma tomografia computadorizada (TC) de crânio (Figura 1), que mostrou hematomas subdural agudo e intraparenquimatoso, com desvio das estruturas de linha média maior que $5 \mathrm{~mm}$. Foi realizado um procedimento cirúrgico com remoção dos hematomas e hemicraniectomia frontotemporo-pariental descompressiva esquerda

(Figuras 2A e B).
Após a abertura da dura-máter em "C" observou-se cérebro tenso. O flap ósseo foi alojado temporariamente no tecido subcutâneo em hipocôndrio esquerdo (Figura 2C).

Cerca de 20 dias após o procedimento, a paciente já se encontrava estável, consciente, interagindo, apresentando uma melhora significativa do quadro clinico, apesar de hemiparesia grau 2 em dimidio direito. Com a estabilização do quadro da paciente $(E C G=15)$ foi realizada então cranioplastia com reposição do flap ósseo que estava armazenado no tecido subcutâneo abdominal (Figura 2D). A paciente evoluiu bem, recebendo alta três dias após a segunda cirurgia.
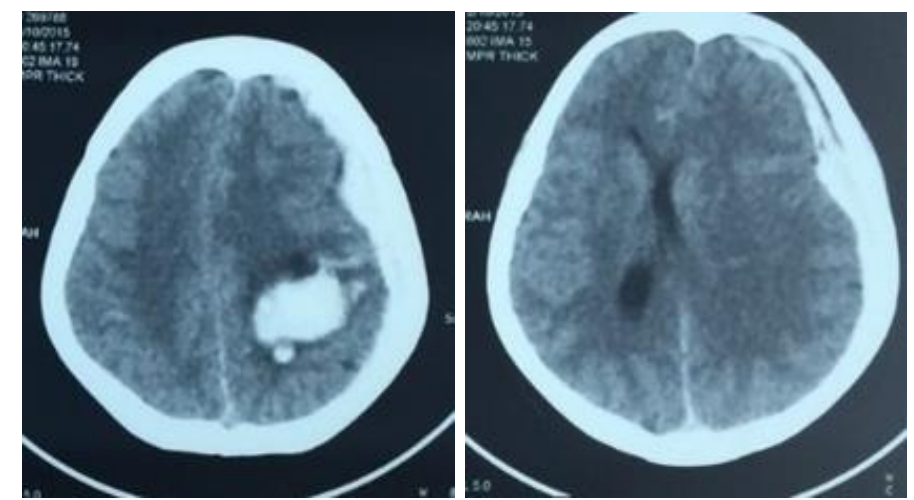

Figura 1. Corte transversal em TC de crânio, evidenciando hematoma subdural à esquerda com indícios de agudização, bem como desvio de linha média e hematoma intraparenquimatoso à esquerda.

\section{Comentários}

Na avaliação do paciente com TCE agudo, a TC é o exame de escolha, podendo ser realizada em duas fases: sem e com contraste. Esta última contribui muito no esclarecimento diagnóstico de doenças em que há quebra da barreira hematoencefálica. A TC sempre deve ser avaliada em três janelas: avaliação do sistema encefálico, detecção de hemorragias hiperdensas e avaliação óssea para garantir a visualização de pequenos hematomas. $^{4,5}$

Entre os sinais sugestivos de HIC encontrados na $\mathrm{TC}$, podemos citar o hematoma intracraniano com efeito de massa e grande desvio das estruturas da linha média, Desaparecimento dos ventrículos laterais e do terceiro ventrículo, dilatação do sistema ventricular (principalmente se acompanhado de hipodensidade ao redor), desaparecimento das cisternas perimesencefálicas e visualização de herniações intracranianas também são características encontradas no TC associados na HIC. ${ }^{2,5}$

Nos casos em que a HIC é decorrente de hemorragias, a TC apresenta como achados característicos o extravasamento de sangue, observado como áreas de hiperdensidade e, para evitar que pequenos sangramentos passem despercebidos, recomenda-se TC com cortes finos, exame que contribui diretamente como fator prognóstico positivo quando realizado 
precocemente em pacientes com rebaixamento do nível de consciência. ${ }^{5,6}$

$\mathrm{Na}$ TC desta paciente observam-se achados de hematoma subdural agudo: coleção hiperatenuante côncavo-convexa, com sua face côncava moldando-se à convexidade cortical, foice cerebelar ou tenta cerebelar e de hematoma intraparenquimatoso: achados de lesões densas na região intracerebral por rompimento de vasos intraparenquimatoso, com ou sem halo hipodenso circunjacente (edema).

A paciente apresentava fatores considerados predisponentes de pior prognóstico, tais como: HIC, ECG inferior a 9, idade maior do que 40 anos, volumoso hematoma em TC no dia de sua admissão e desvio de linha média $>5 \mathrm{~mm}^{5,7}$, o que justificou o internamento para realização da craniectomia descompressiva de emergência. ${ }^{8}$ Apesar desses fatores de mal progáśstico, a paciente teve boa
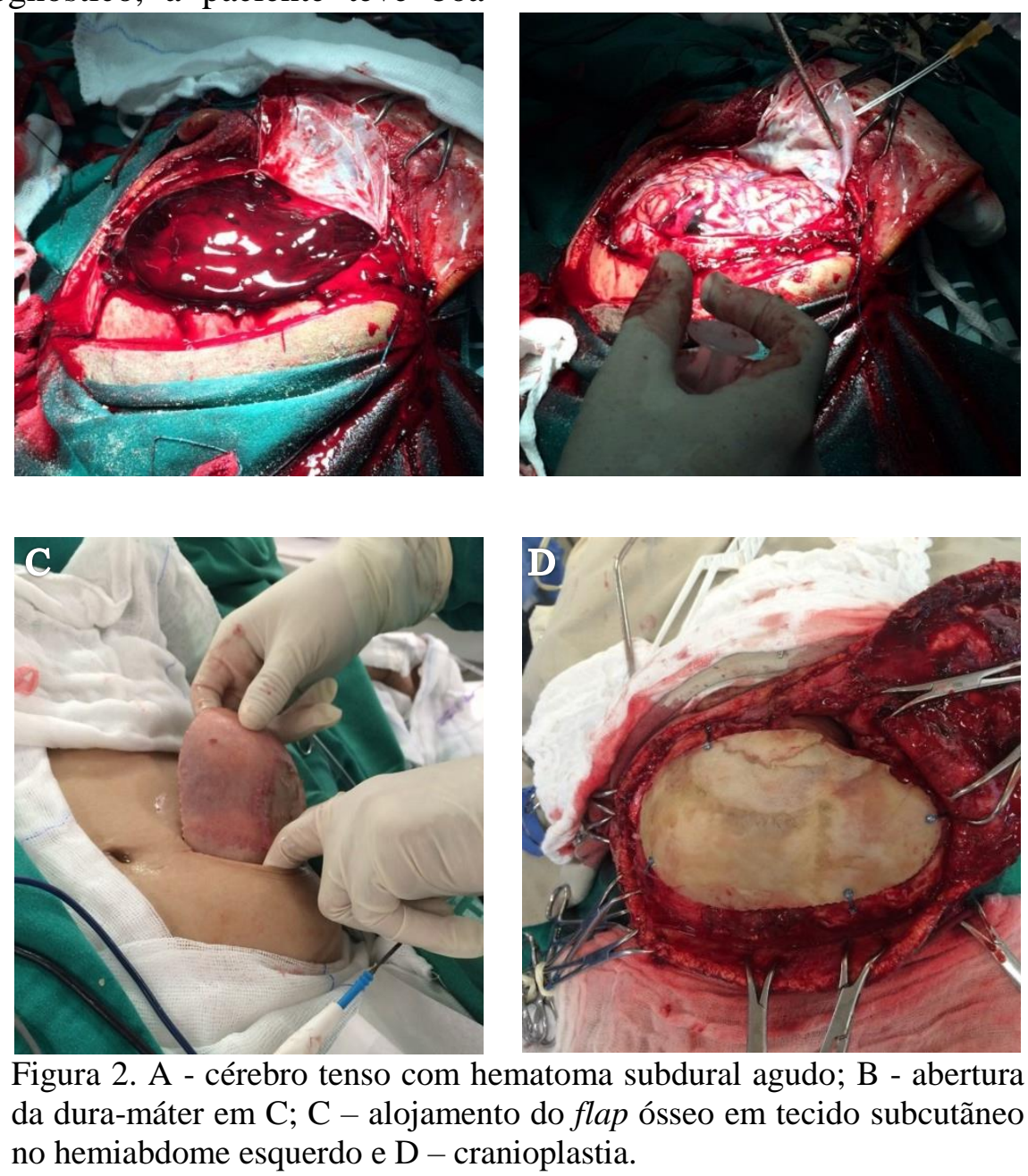

Figura 2. A - cérebro tenso com hematoma subdural agudo; B - abertura da dura-máter em C; $\mathrm{C}$ - alojamento do flap ósseo em tecido subcutãneo no hemiabdome esquerdo e $\mathrm{D}$ - cranioplastia.

\section{Referências}

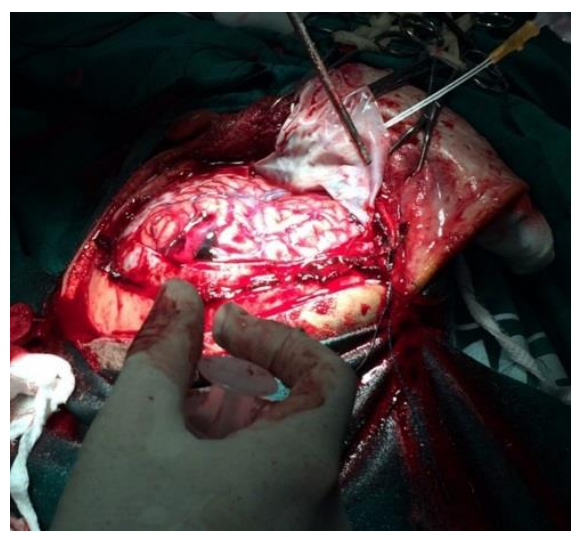

evolução no pós-operatório de ambas as cirurgias. Pode-se atribuir tal sucesso ao tempo hábil no reconhecimento e condução da HIC e, também, ao fato da cranioplastia ter sido realizada de modo precoce, reduzindo assim o risco de complicações comuns, bem como melhorando as funções do córtex cerebral subjacente. ${ }^{5,6,9} \mathrm{O}$ uso do flap ósseo preservado em subcutâneo permite não só a realização da cranioplastia precoce como também a redução de custo quando comparado a outros materiais. A importância da cranioplastia precoce se deve à Síndrome do Trefinado, que consiste em déficits cognitivos e/ou motores causados por um tempo elevado entre a craniotomia e a cranioplastia. Apesar dos sintomas regredirem parcialmente após uma cranioplastia tardia, as sequelas neurológicas são de consequência vital para a qualidade de vida e produtividade dos pacientes e seus familiares. ${ }^{9,10}$ 
1. Ashayeri, K., M. Jackson, E., Huang, J., Brem, H., \& R. Gordon, C. (2016). Syndrome of the Trephined. Neurosurgery, 79(4), 525-534.

2. Carlotti JCG, Colli BO, Dias LAA (1998) Hipertensão intracraniana. Medicina, Ribeirão Preto, 31: 552-562.

3. Rossini, Z., Nicolosi, F., Kolias, A., Hutchinson, P., De Sanctis, P., \& Servadei, F. (2019). The History of Decompressive Craniectomy in Traumatic Brain Injury. Frontiers In Neurology, 10. (458), 1-9.

4. Gattás GS (2011) Imagem no traumatismo craniano / Imaging of traumatic brain injury. Rev Med 90(4):157-68.

5. Carroll, J., Lavine, S., \& Meyers, P. (2017). Imaging of Subdural Hematomas. Neurosurgery Clinics Of North America, 28(2) 1-25.

6. Fountain, D., Kolias, A., Lecky, F., Bouamra, O., Lawrence, T., \& Adams, H. et al. (2017). Survival
Trends After Surgery for Acute Subdural Hematoma in Adults Over a 20-year Period. Annals Of Surgery, 265(3), 590-596.

7. Grille, P., \& Tommasino, N. (2015). Decompressive craniectomy in severe traumatic brain injury: prognostic factors and complications. Revista Brasileira de Terapia Intensiva, 27(2) 113-118.

8. Moon, J., \& Hyun, D. (2017). Decompressive Craniectomy in Traumatic Brain Injury: A Review Article. Korean Journal of Neurotrauma, 13(1), 1-8.

9. Gopalakrishnan, M., Shanbhag, N., Shukla, D., Konar, S., Bhat, D., \& Devi, B. (2018). Complications of Decompressive Craniectomy. Frontiers In Neurology, 9(977) 1-8.

10. Ashayeri, K., M. Jackson, E., Huang, J., Brem, H., \& R. Gordon, C. (2016). Syndrome of the Trephined. Neurosurgery, $\quad 79(4), \quad 525-534$. 